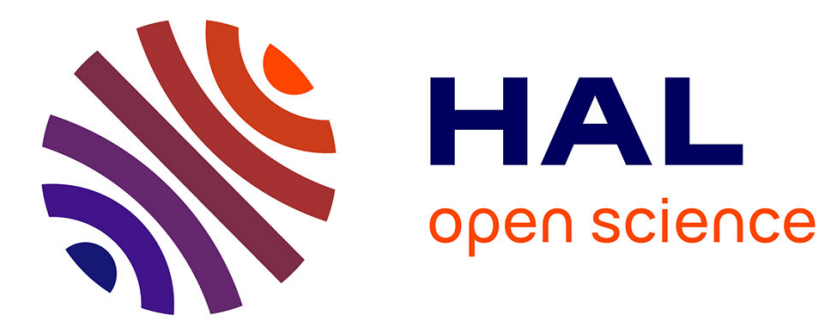

\title{
Electron paramagnetic resonance image reconstruction with total variation and curvelets regularization
}

\author{
Sylvain Durand, Yves-Michel Frapart, Maud Kerebel
}

\section{To cite this version:}

Sylvain Durand, Yves-Michel Frapart, Maud Kerebel. Electron paramagnetic resonance image reconstruction with total variation and curvelets regularization. 2017. hal-01419832v2

\section{HAL Id: hal-01419832 \\ https://hal.science/hal-01419832v2}

Preprint submitted on 8 Feb 2017

HAL is a multi-disciplinary open access archive for the deposit and dissemination of scientific research documents, whether they are published or not. The documents may come from teaching and research institutions in France or abroad, or from public or private research centers.
L'archive ouverte pluridisciplinaire HAL, est destinée au dépôt et à la diffusion de documents scientifiques de niveau recherche, publiés ou non, émanant des établissements d'enseignement et de recherche français ou étrangers, des laboratoires publics ou privés. 


\title{
Electron paramagnetic resonance image reconstruction with total variation and curvelets regularization
}

\author{
Sylvain Durand ${ }^{1}$, Yves-Michel Frapart ${ }^{2}$ and \\ Maud KereBeL ${ }^{1,2}$ \\ ${ }^{1}$ Laboratoire de Mathématiques Appliquées à Paris 5 - UMR CNRS 8045, \\ Université Paris Descartes, Sorbonne Paris Cité \\ ${ }^{2}$ Laboratoire de Chimie et Biochimie Pharmacologiques et Toxicologiques - \\ UMR CNRS 8601, FR3657 CICB, FR3443 RENARD, Université Paris \\ Descartes, Sorbonne Paris Cité \\ E-mail: sylvain.durand@parisdescartes.fr, \\ yves.frapart@parisdescartes.fr and maud.kerebel@parisdescartes.fr
}

\begin{abstract}
Spatial electron paramagnetic resonance imaging (EPRI) is a recent method to localize and characterize free radicals in vivo or in vitro, leading to applications in material and biomedical sciences. To improve the quality of the reconstruction obtained by EPRI, a variational method is proposed to inverse the image formation model. It is based on a leastsquare data-fidelity term and the total variation and Besov seminorm for the regularization term. To fully comprehend the Besov seminorm, an implementation using the curvelet transform and the $L^{1}$ norm enforcing the sparsity is proposed. It allows our model to reconstruct both image where acquisition information are missing and image with details in textured areas, thus opening possibilities to reduce acquisition times. To implement the minimization problem using the algorithm developed by Chambolle and Pock, a thorough analysis of the direct model is undertaken and the latter is inverted while avoiding the use of filtered backprojection (FBP) and of non-uniform Fourier transform. Numerical experiments are carried out on simulated data, where the proposed model outperforms both visually and quantitatively the classical model using deconvolution and FBP. Improved reconstructions on real data, acquired on an irradiated distal phalanx, were successfully obtained.
\end{abstract}




\section{Introduction}

Electron paramagnetic resonance (EPR) is a spectroscopy method that characterizes and quantifies [43] molecules with paramagnetic properties, such as free radicals or transition metals. Fields of application are various and englobe, among others, pharmacy [29], chemistry [23], medicine [26] or dermatology [33].

Electron paramagnetic resonance imaging (EPRI) is a technology that maps the spatial distribution of a paramagnetic species [16], making it part of the molecular imaging technologies, by opposition and complementarity to anatomic and functional imaging. Fields of application include oximetry [41], cardiology [28], drug release [8], geology [7], redox [19] or batteries [13]. Similarly to EPR, the sample is submitted to a specific magnetic field that triggers the resonance phenomenon of the electronic spins of the paramagnetic molecule. In order to retrieve the spatial repartition of the latter, a magnetic field gradient is added. Spectroscopy acquisitions are conducted for each orientation of the gradient, yielding a variety of spectrum. The set of raw data obtained by this acquisition process is called a sinogram, and the object of this paper is to retrieve from the sinogram the image featuring the spatial repartition of the paramagnetic species.

The mathematical modelisation of the process turning an image $u$ into a sinogram $S$ is the convolution

$$
S=R u * h
$$

Quantity $h$ stands for the spectrum of the paramagnetic species and $R u$ for the Radon transform of $u$, first introduced a hundred years ago by Johann Radon [35]. Inverting the Radon transform is an ill-posed problem [3] and has been the subject of many papers.

The classical approach for EPRI reconstruction is the filtered backprojection (FBP), thoroughly explained for computed tomography (CT) [22], associated with a deconvolution step. It is the choice made by commercial software for EPRI reconstruction such as Xepr [1]. Even though the FBP is a simple and time-efficient method [21], it admits some major drawbacks [32]: FBP requires equally-spaced acquisition angles and is not robust to a low number of acquisition angles. The current development of EPRI goes toward in vivo imaging and clinical applications [14] [26]: because acquisition times are significantly long in EPRI, it may be interesting to decrease the sampling, and it is not possible due to the traditional post processing of the data. 
Improvements of the FBP have been proposed specifically for EPRI [5]. Unfortunately, the issues of backprojection, deconvolution, deblurring and denoising are treated as separate problems, thus leading to approximation errors that add to each other.

To consider the reconstruction as a whole issue instead of the sum of subproblems, variational methods are well suited. Tikhonov and entropy-based regularization have been proposed in [24] and [40], in which the desired image $u$ is retrieved from the data $S$ by solving

$$
\underset{u}{\arg \min } \frac{1}{2}\|C u-S\|_{2}^{2}+\lambda J(u)
$$

where $C u=R u * h$ models the response of an EPRI device and $S$ is the raw data, or sinogram. The second term $J(u)$ corresponds either to the Tikhonov regularization [39] or to the Shannon entropy [24], ponderated by a coefficient $\lambda$.

More recently, [25] has proposed an objective function making most of the sparsity induced by the $L^{1}$ norm. It retrieves the image $u$ solution of

$$
\underset{u}{\arg \min } \frac{1}{2}\|C u-S\|_{2}^{2}+\lambda_{1}\|u\|_{1}+\lambda_{2} \operatorname{TV}(u)
$$

where the second regularization term $\operatorname{TV}(u) \simeq\|\nabla u\|_{1}$ stands for the total variation of $u$. They obtain satisfying numerical results, although their minimization scheme is not formally proven and may lead to false convergence results. Indeed, [25] solves problem (3) by minimizing the objective function with alternatively applying one step of FISTA algorithm to the objective function with $\lambda_{2}=0$ and then applying the proximal operator of $\lambda_{2} \mathrm{TV}$.

Another reference [34] uses the total variation regularization for EPRI by solving

$$
\min \operatorname{TV}(u) \quad \text { s.t. } \quad S=C u
$$

In this model, the hard constraint on the data consistency does not take the noise created by the device into account. The minimization part is ensured with a gradient descent applied to the objective function smoothed where the $L^{1}$ norm is not differentiable.

However, huge progress has been made recently in the field of optimization and new algorithms have been developed to solve this kind of variational problems. We propose a new regularization, especially adapted for images with textures, and a thorough analysis of the direct problem which allow us to minimize efficiently the objective function. 
Our first objective function is a simpler version of (3) with only the total variation as regularization

$$
\underset{u}{\arg \min } \frac{1}{2}\|C u-S\|_{2}^{2}+\lambda \mathrm{TV}(u)
$$

Because this simple regularization is not subtle enough to deal with images with textures, we propose a second model with another regularization term: a combination of the total variation and a ponderated $L^{1}$ norm on the curvelets coefficients

$$
\underset{u=u_{1}+u_{2}}{\arg \min }\left\|C\left(u_{1}+u_{2}\right)-S\right\|_{2}^{2}+\lambda_{1} \operatorname{TV}\left(u_{1}\right)+\lambda_{2}\left\|W u_{2}\right\|_{1}
$$

where the additional regularization term has the interesting property to retrieve the texture in the reconstructed image.

To efficiently solve problems (5) and (6), we undergo an analysis of the dataconsistency term which highlights the presence of $C^{*} C$ as a Toeplitz operator [15]. It leads to acceleration properties that allow us to minimize the objective functions with the algorithm recently developed by Chambolle and Pock [10]. Tests are conducted on both simulated data and real data. Simulations are processed on the Shepp-Logan phantom, both the original phantom and a textured phantom. Real data are acquired on an irradiated distal phalanx. The irradiation map retrieved with EPRI admits details whose existence is confirmed by a comparison with an anatomic map of the bone density performed by a CT-scanner.

\section{Model of spatial EPRI}

\subsection{Direct model}

In the case of EPR spectroscopy, the magnetic field $B$ inside the acquisition cavity is spatially uniform and varies over time. In the case of EPRI, an orientated field gradient $\mu \mathbf{e}_{\theta}$ is added inside the cavity, where $\mathbf{e}_{\theta}=$ $\left(\cos \theta_{1} \cos \theta_{2}, \sin \theta_{1} \cos \theta_{2}, \sin \theta_{2}\right)$ represents the unit vector whose direction is characterized in spherical coordinates by the angles $\theta=\left(\theta_{1}, \theta_{2}\right)$. At the point $\mathbf{x} \in \mathbb{R}^{3}$, the total magnetic field is the sum of the field $B$ and the field gradient

$$
B+\mu \mathbf{e}_{\theta} \cdot \mathbf{x}
$$

The addition of a microwave causes the resonance phenomenon of the free electrons. Due to the quantization of energy levels, the resonance property 
happens where the total magnetic field is equal to the resonance magnetic field $B_{\text {res }}$. However, it is impossible to create a magnetic field with absolute accuracy, and even in that case the precision would theoretically be limited by the uncertainty principle. Therefore the resonance can happen shortly before of after the expected resonance field $B_{\text {res }}$. The repartition of those non-exact resonances has the shape of the distribution $g_{0}$, which is a mixture of gaussian and/or lorentzian distributions and characterizes the paramagnetic species.

To remove some noise from the acquisition data, the energy absorbed by the electronic transitions is measured through the process of amplitude modulation. It implies that the distribution of the inexact resonance has the shape of $h_{0}$ the derivative of $g_{0}$. At spatial position $\mathbf{x} \in \mathbb{R}^{3}$, the absorbed energy is directly proportional to the quantity $v(\mathbf{x})$ of the studied paramagnetic species

$$
E_{\mathrm{abs}}(\mathbf{x})=v(x) h_{0}\left(B+\mu \mathbf{e}_{\theta} \cdot \mathbf{x}\right)
$$

However, the measurement takes place all over the cavity and not only at position $\mathbf{x}$. Therefore the accessible raw data at magnetic field $B$ and field gradient orientation $\theta$ is the total amount of absorbed energy, whose sum over every spatial position gives the sinogram

$$
S_{0}(B, \theta)=\int_{\mathbb{R}^{3}} v(\mathbf{x}) h_{0}\left(B+\mu \mathbf{e}_{\theta} \cdot \mathbf{x}\right) d \mathbf{x}
$$

Any spatial position $\mathbf{x}$ can be uniquely decomposed into $\mathbf{x}=y \mathbf{e}_{\theta}+\mathbf{z}$ with $\mathbf{z} \in H_{\theta}$ where $H_{\theta}$ is the hyperplane passing through the point of spherical coordinates $\left(y, \theta_{1}, \theta_{2}\right)$ and orthogonal to $\mathbf{e}_{\theta}$. It leads to the following expression of the sinogram

$$
S_{0}(B, \theta)=\int_{\mathbb{R}}\left(\int_{H_{\theta}} v\left(y \mathbf{e}_{\theta}+\mathbf{z}\right) d \mathbf{z}\right) h_{0}(B+\mu y) d y
$$

because $y=\mathbf{e}_{\theta} \cdot \mathbf{x}$. Here appears the 3D Radon transform of $v$ at angle $\theta$

$$
R_{\theta} v(y)=\int_{H_{\theta}} v\left(y \mathbf{e}_{\theta}+\mathbf{z}\right) d \mathbf{z}
$$

which yields

$$
S_{0}(B, \theta)=\int_{\mathbb{R}} R_{\theta} v(y) h_{0}\left(-\mu\left(-\frac{B}{\mu}-y\right)\right) d y
$$


By considering both sinogram and spectrum in spatial position instead of magnetic field by $S(x, \theta)=S_{0}(-\mu x, \theta)$ and $h(x)=h_{0}(-\mu x)$, equation (12) yields the convolution product

$$
S(x, \theta)=\left(R_{\theta} v * h\right)(x) \stackrel{\text { def }}{=} \int_{\mathbb{R}} R_{\theta} v(y) h(x-y) d y
$$

Similarly to [31], [39] and [4], we assume that the data is corrupted with an additive independant gaussian white noise denoted $n$, thus yielding the direct model of spatial EPRI acquisition

$$
S(x, \theta)=\left(h * R_{\theta} v\right)(x)+n(x, \theta)
$$

However, EPRI acquisitions are not always made in 3D, but also in 1D and in $2 \mathrm{D}$. For $2 \mathrm{D}$ acquisition, the angle $\theta_{2}$ is set to 0 , and the field gradient is supported by the vector $\mathbf{e}_{\theta}=(\cos \theta, \sin \theta)$ in the plane $\theta_{2}=0$. The retrieved $2 \mathrm{D}$ image $u$ is the projection of the $3 \mathrm{D}$ image $\mathrm{v}$ on the plane $\theta_{2}=0$. We have indeed

$$
R_{\theta}^{2 d} u=R_{(\theta, 0)}^{3 d} v
$$

where $R^{2 d}$ represents the 2D Radon transform and $R^{3 d}$ represents the 3D Radon transform. All our simulation and experiences are in 2D, that's why the following work considers only the 2D Radon transform, still denoted $R_{\theta}$ with $\theta$ a single angle, and the 2D image $u$. The following work can however be generalized to 3D reconstructions.

\subsection{Data-fidelity term}

In order to retrieve the $2 \mathrm{D}$ image $u$ from the data $S$ whose relation is described in equation (14), we employ the maximum a posteriori method. It requires the definition of a data-difelity term and a regularization term.

The $L^{2}$ norm is the log-likelihood estimate to remove the independent white gaussian noise from the data, thus yielding the data-fidelity term

$$
F(u)=\frac{1}{2}\left\|h * R_{\theta} u-S\right\|_{2}^{2}
$$

For simplicity, we will rewrite this equation in terms of the Fourier transform of $u$ which is defined, for $u \in L^{1}(\mathbb{R})$, by $\widehat{u}(\xi)=\int u(x) e^{-2 i \pi x \cdot \xi} d x$. By using the associated Plancherel equality, we obtain

$$
F(u)=\frac{1}{2}\left\|\widehat{h} \cdot \widehat{R_{\theta} u}-\widehat{S}\right\|_{2}^{2} .
$$


Projection-slice theorem [11] states that, for all $\xi \in \mathbb{R}$ and $\theta \in[0, \pi]$,

$$
\widehat{R_{\theta} u}(\xi)=\widehat{u}\left(\xi \mathbf{e}_{\theta}\right)
$$

with $\mathbf{e}_{\theta}=(\cos \theta, \sin \theta)$. It means that, for every acquisition angle $\theta$, the 1 D Fourier transform of the Radon transform of image $u$ at angle $\theta$ is equal to the 2D Fourier transform of image $u$ evaluated on the line carried by $\mathbf{e}_{\theta}$. Data-fidelity term (16) has thus become

$$
F(u)=\frac{1}{2}\|A u-d\|_{2}^{2}
$$

with $d: \stackrel{\text { def }}{=} \widehat{S}$ and $A$ being the linear operator in variable $u$

$$
A u(\xi, \theta): \stackrel{\text { def }}{=} \widehat{h}(\xi) \widehat{u}\left(\xi \mathbf{e}_{\theta}\right)
$$

The Radon transform is typically defined on the Schwartz space $S\left(\mathbb{R}^{2}\right)$ and the same applies for $A$. The spectrum $h$ that is used in its definition is however the derivative of a function $g$ that is a finite linear combination of Gaussian and Lorentzian functions. The Fourier transforms $\widehat{h}$ and $\widehat{g}$ have therefore fast decay. Contrary to the Radon transform alone, the mapping $A$ can therefore be easily extended onto $L^{2}\left(\mathbb{R}^{2}\right)$ by continuity, thus allowing to formally define its adjoint.

Proposition 1 The operator $A$ defined on $S\left(\mathbb{R}^{2}\right)$ admits a unique continuous extension from $L^{2}\left(\mathbb{R}^{2}\right)$ to $L^{2}(\mathbb{R} \times[0, \pi])$.

Proof. Observe that, since $\widehat{h}$ and $\widehat{g}$ are bounded, there exists $c>0$ such that for every $\xi \in \mathbb{R}$

$$
|\widehat{h}(\xi)|^{2}=4 \pi^{2} \xi^{2}|\widehat{g}(\xi)|^{2} \leqslant \min \left(4 \pi^{2} \xi^{2}\|\widehat{g}\|_{\infty}^{2},\|\widehat{h}\|_{\infty}^{2}\right) \leqslant c|\xi| .
$$

Hence, for every $u \in S\left(\mathbb{R}^{n}\right)$

$$
\begin{aligned}
\int_{0}^{\pi} \int_{\mathbb{R}}|A u(\xi, \theta)|^{2} d \xi d \theta & =\int_{0}^{\pi} \int_{\mathbb{R}}\left|\widehat{h}(\xi) \widehat{u}\left(\xi \mathbf{e}_{\theta}\right)\right|^{2} d \xi d \theta \\
& \leqslant c \int_{0}^{\pi} \int_{\mathbb{R}}|\xi|\left|\widehat{u}\left(\xi \mathbf{e}_{\theta}\right)\right|^{2} d \xi d \theta \\
& =c \int_{\mathbb{R}^{2}}|\widehat{u}(\omega)|^{2} d \omega \\
& =c \int_{\mathbb{R}^{2}}|u(x)|^{2} d x
\end{aligned}
$$

The conclusion follows from the density of $S\left(\mathbb{R}^{2}\right)$ in $L^{2}\left(\mathbb{R}^{2}\right)$. 
The following proposition gives a simple way to compute the operator $A^{*} A$, where $A^{*}$ stands for the adjoint operator of $A$. This expression appears in the minimization algorithms proposed thereafter.

Proposition 2 For every $u \in L^{2}\left(\mathbb{R}^{2}\right)$

$$
A^{*} A u=k * u
$$

where the point spread function $k$ is defined by

$$
k(x): \stackrel{\text { def }}{=} \int_{0}^{\pi} \int_{\mathbb{R}}|\widehat{h}(\xi)|^{2} e^{2 i \pi \xi x \cdot \mathbf{e}_{\theta}} d \xi d \theta, \quad \forall x \in \mathbb{R}^{2}
$$

Proof. Let us first compute the adjoint operator $A^{*}: L^{2}(\mathbb{R} \times[0, \pi]) \rightarrow L^{2}\left(\mathbb{R}^{2}\right)$ that is well defined thanks to the continuous extension of proposition 1 . For all $u \in L^{1}\left(\mathbb{R}^{2}\right) \cap L^{2}\left(\mathbb{R}^{2}\right)$ and $v \in L^{2}\left(\mathbb{R}^{2}\right)$, we have

$$
\int_{\mathbb{R}^{2}} \int_{0}^{\pi} \int_{\mathbb{R}}|u(x) \widehat{h}(\xi) v(\xi, \theta)| d \xi d \theta d x \leq\|u\|_{L^{1}\left(\mathbb{R}^{2}\right)} \sqrt{\pi}\|\widehat{h}\|_{L^{2}(\mathbb{R})}\|v\|_{L^{2}(\mathbb{R} \times[0, \pi])}<\infty
$$

We can therefore apply Fubini's theorem in order to show that

$$
\begin{aligned}
\langle A u, v\rangle & =\int_{0}^{\pi} \int_{\mathbb{R}} A u(\xi, \theta) \overline{v(\xi, \theta)} d \xi d \theta \\
& =\int_{0}^{\pi} \int_{\mathbb{R}} \widehat{h}(\xi)\left(\int_{\mathbb{R}^{2}} u(x) e^{-2 i \pi \xi x \cdot \mathbf{e}_{\theta}} d x\right) \overline{v(\xi, \theta)} d \xi d \theta \\
& =\int_{\mathbb{R}^{2}} u(x)\left(\int_{0}^{\pi} \int_{\mathbb{R}} \widehat{h}(\xi) \overline{v(\xi, \theta)} e^{-2 i \pi \xi x \cdot \mathbf{e}_{\theta}} d \xi d \theta\right) d x
\end{aligned}
$$

where $\overline{v(\xi, \theta)}$ denotes the complex conjugate of $v(\xi, \theta)$. Identifying the latter with $\left\langle u, A^{*} v\right\rangle$, for all $u \in L^{1}\left(\mathbb{R}^{2}\right) \cap L^{2}\left(\mathbb{R}^{2}\right)$ which is dense in $L^{2}\left(\mathbb{R}^{2}\right)$, yields the expression of the adjoint operator

$$
A^{*} v(x)=\int_{0}^{\pi} \int_{\mathbb{R}} \overline{\widehat{h}(\xi)} v(\xi, \theta) e^{2 i \pi \xi x \cdot \mathbf{e}_{\theta}} d \xi d \theta
$$

and allows to compute $A^{*} A u$. We have indeed, for all $u \in L^{1}\left(\mathbb{R}^{2}\right) \cap L^{2}\left(\mathbb{R}^{2}\right)$

$$
\begin{aligned}
\left(A^{*} A u\right)(x) & =\int_{0}^{\pi} \int_{\mathbb{R}} \overline{\widehat{h}(\xi)} A u(\xi, \theta) e^{2 i \pi \xi x \cdot \mathbf{e}_{\theta}} d \xi d \theta \\
& =\int_{0}^{\pi} \int_{\mathbb{R}} \widehat{\widehat{h}(\xi)} \widehat{h}(\xi)\left(\int_{\mathbb{R}^{2}} u(y) e^{-2 i \pi \xi y \cdot \mathbf{e}_{\theta}} d y\right) e^{2 i \pi \xi x \cdot \mathbf{e}_{\theta}} d \xi d \theta \\
& =\int_{\mathbb{R}^{2}} u(y)\left(\int_{0}^{\pi} \int_{\mathbb{R}}|\widehat{h}(\xi)|^{2} e^{2 i \pi \xi(x-y) \cdot \mathbf{e}_{\theta}} d \xi d \theta\right) d y \\
& =\int_{\mathbb{R}^{2}} u(y) k(x-y) d y \\
& =(u * k)(x)
\end{aligned}
$$


where the third equality is a consequence of Fubini's theorem again, since

$$
\int_{\mathbb{R}^{2}} \int_{0}^{\pi} \int_{\mathbb{R}}\left|u(y) \widehat{h}(\xi)^{2}\right| d \xi d \theta d y \leq \pi\|u\|_{L^{1}\left(\mathbb{R}^{2}\right)}\|\widehat{h}\|_{L^{2}(\mathbb{R})}<\infty
$$

It remains to show that $(21)$ is still satisfied when $u \in L^{2}\left(\mathbb{R}^{2}\right)$. Observe that $|\widehat{h}|$ is even because $h$ is real valued. We infer that

$$
\begin{aligned}
k(x) & =\int_{-\pi}^{\pi} \int_{0}^{\infty}|\widehat{h}(\xi)|^{2} e^{2 i \pi \xi x \cdot \mathbf{e}_{\theta}} d \xi d \theta \\
& =\int_{-\pi}^{\pi} \int_{0}^{\infty} \xi|\widehat{g}(\xi) \widehat{h}(\xi)| e^{2 i \pi \xi x \cdot \mathbf{e}_{\theta}} d \xi d \theta \\
& =\int_{\mathbb{R}^{2}}|\widehat{g}(|\omega|) \widehat{h}(|\omega|)| e^{2 i \pi x \cdot \omega} d \omega
\end{aligned}
$$

Since $\omega \mapsto|\widehat{g}(|\omega|) \widehat{h}(|\omega|)|$ belongs to $L^{2}\left(\mathbb{R}^{2}\right)$, its inverse Fourier transform $k$ belongs to $L^{2}\left(\mathbb{R}^{2}\right)$ as well, and the mapping $u \mapsto k * u$ can be extended into a continuous operator from $L^{2}\left(\mathbb{R}^{2}\right)$ to $L^{\infty}\left(\mathbb{R}^{2}\right)$. On the other hand, $A^{*} A$ is continuous from $L^{2}\left(\mathbb{R}^{2}\right)$ to itself, and we can conclude the proof by a density argument.

$\diamond$

\subsection{Regularization term}

1.3.1. Total variation A first choice for the regularization term is the total variation

$$
\operatorname{TV}(u)=\sup \left\{\int_{\mathbb{R}^{2}} u \operatorname{div} \phi\left|\phi \in \mathcal{C}_{c}^{\infty},\right| \phi(x) \mid \leqslant 1\right\}
$$

whose interest regarding computer vision has first been discussed in [36]. The expression for a discrete or $W^{1,1}$ image $u$ is

$$
\mathrm{TV}(u)=\int|\nabla u|=\|\nabla u\|_{1}
$$

An accurate inversion of equation (14) is obtained by solving

$$
\bar{u}=\underset{u}{\arg \min } F(u)+\lambda \operatorname{TV}(u)
$$

where $\lambda$ is a trade-off parameter to balance the effect of the total variation regularization with the data-fidelity term $F$, and allows to choose the remaining level of noise.

The main advantages of this regularization choice are the sharpness of the reconstructed images and its ability to reconstruct images from incomplete data. 
It also tends to recreate images that are piecewise constant, due to the enforced sparsity of the $L^{1}$ norm applied to the gradient. Therefore, the total variation regularization is well adapted to images without oscillations.

However, textured areas suffer from the use of total variation regularization and tends to become homogeneous with this reconstruction. This is why another prior is presented along with it.

1.3.2. Total variation and curvelets The second regularization model harnesses the idea of [30] to decompose an image $u$ into its cartoon part $u_{1}$ and its textured part $u_{2}$. The reconstructed image $u$ is then the sum of the two images $u_{1}$ and $u_{2}$

$$
u=u_{1}+u_{2}
$$

While it is generally acknowledged that the total variation allows to efficiently model cartoon images, several criteria, nearing more or less the dual norm of the total variation, have been proposed in order to characterize the texture component, or more precisely the oscillating component. Among the most notable ones, we can quote $\operatorname{div}(B M O)$ [30], $\operatorname{div}\left(L^{\infty}\right)$ [30], the homogeneous Besov norm $\dot{B}_{\infty, \infty}^{-1}[30]$ and the homogeneous Sobolev norm $\dot{H}^{-1}[42]$.

Contrarily to [42] or [6], our objective is not to obtain the best decomposition into a cartoon and a texture part, but to retrieve the sum $u_{1}+u_{2}$ from the noisy data. We propose therefore to use a criterion that allows to separate efficiently the texture and the noise and that is based, to this end, on a sparse representation of the texture. To our knowledge, the closest model to ours was introduced by Frühauf, Pontow and Scherzer [18] who characterized the textured part $u_{2}$ with the $L^{1}$ norm of its weighted curvelet transform $W u_{2}=\left(w_{j}\left\langle u_{2}, \phi_{j, k}\right\rangle\right)_{j, k}$, the index $j$ being the scale parameter and $k$ standing for the translation and direction parameters of the curvelet $\phi_{j, k}$. However, unlike [18], we set $w_{j}=2^{-\frac{n+2}{2} j}$ where $n$ is the dimension of the space on which the image is defined. Hence, for 2D images, we have

$$
\left\|W u_{2}\right\|_{1}=\sum_{j, k} 4^{-j}\left|\left\langle u_{2}, \phi_{j, k}\right\rangle\right|
$$

If the curvelet frame is changed into a wavelet basis, it is equivalent to the homogeneous Besov $\dot{B}_{1,1}^{-1}$ norm [30] that is close to the Sobolev $\dot{W}_{1}^{-1}$ norm.

The reconstructed image $\bar{u}$ is thus solution of the problem

$$
\bar{u}=\underset{u=u_{1}+u_{2}}{\arg \min }\left(F\left(u_{1}+u_{2}\right)+\lambda_{1} \operatorname{TV}\left(u_{1}\right)+\lambda_{2}\left\|W u_{2}\right\|_{1}\right)
$$


where $\lambda_{1}$ and $\lambda_{2}$ are parameters associated with each prior. They can be adapted to the level of cartoon or textured effect desired in the image $\bar{u}$.

\section{Minimization algorithm}

\subsection{First-order primal-dual algorithm}

Chambolle and Pock [10] give an efficient iterative algorithm to minimize problems of the form

$$
\underset{u}{\arg \min } f(u)+g(K u)
$$

with $f$ and $g$ proper, positive, lower semi-continuous and convex functions, and $K$ a continuous linear operator. The minimization algorithm consists of the steps

Initialization Initialize $\sigma, \tau>0, u^{0} \in \mathbb{R}^{n}, p^{0} \in \mathbb{R}^{n} \times \mathbb{R}^{n}$ and set $v^{0}=u^{0}$.

ITERATIONS For $k \geqslant 0$, iterate

(a) $p^{k+1}=\left(I+\sigma \partial g^{\star}\right)^{-1}\left(p^{k}+\sigma K v^{k}\right)$

(b) $u^{k+1}=(I+\tau \partial f)^{-1}\left(u^{k}-\tau K^{*} p^{k+1}\right)$

(c) $v^{k+1}=2 u^{k+1}-u^{k}$

where $I$ stands for the identity operator, $\partial f$ the subgradient of $f$ and $g^{\star}$ the conjugate function of $g$, according to the Legendre-Fenchel duality

$$
g^{\star}(u)=\sup _{v}\langle u, v\rangle-g(v)
$$

Step (a) is a gradient ascent in the dual space composed with the proximal operator of $\sigma g^{\star}$. Step (b) is a gradient descent in the primal space composed with the proximal operator of $\tau f$. Step (c) is an acceleration step by extrapolating the point to which apply step (a). Under the condition $\sigma \tau\|K\|^{2}<1$, this algorithm converges to a minimizer of problem (30). For convergence acceleration, those parameters may be modified during the run of the algorithm, mimicking a new minimization problem initialized with the previous $u$ obtained.

By choosing $f(u)=F(u), K u=\lambda \nabla u$ and $g(p)=\|p\|_{1}$ with $p=\left(p_{1}, p_{2}\right)$ the dual variable of $\nabla u$, the primal-dual algorithm converges to a solution of problem (26). By choosing $f\left(u_{1}, u_{2}\right)=F\left(u_{1}+u_{2}\right), K\left(u_{1}, u_{2}\right)=\left(\lambda_{1} \nabla u_{1}, \lambda_{2} W u_{2}\right)$ and $g(p, w)=\|p\|_{1}+\|w\|_{1}$ with $p$ the adjoint variable of $\nabla u_{1}$ and $w$ the adjoint variable of $W u_{2}$, it converges to a solution of problem (29).

Note that by choosing $K(u)=\left(\lambda_{1} \nabla u, \lambda_{2} u\right)$ instead, the algorithm converges to a solution of (3). This method is a more general and flexible method, and 
furthermore the convergence of the Chambolle and Pock algorithm is formally proven in [10].

\subsection{Implementation}

2.2.1. TV model For step (a), the computation of the resolvent $\left(I+\sigma \partial g^{\star}\right)^{-1}$ for $g(p)=\|p\|_{1}$ applied to $\widetilde{p}=p^{k}+\sigma \lambda \nabla v^{n}$ is detailed in [9]. The computation yields

$$
p^{k+1}=\left(I+\sigma \partial G^{*}\right)^{-1}(\widetilde{p}) \Longleftrightarrow p_{i, j}^{k+1}=\frac{\widetilde{p}_{i, j}}{\max \left(1,\left|\tilde{p}_{i, j}\right|\right)}
$$

with $\left|p_{i, j}\right|=\sqrt{\left(p_{1}[i, j]\right)^{2}+\left(p_{2}[i, j]\right)^{2}}$

Step (b) involves $K^{*}(p)=-\lambda \operatorname{div}(p)$ the adjoint operator of $K(u)=\lambda \nabla u$. The computation of the resolvent $(I+\tau \partial F)^{-1}$ for the data-fidelity term $F(u)=$ $\frac{1}{2}\|A u-d\|_{2}^{2}$ applied to $\widetilde{u}=u^{k}+\tau \lambda \operatorname{div} p^{n+1}$ is the solution of

$$
u^{k+1}=\underset{u}{\arg \min } \frac{1}{2}\|u-\tilde{u}\|_{2}^{2}+\tau F(u)
$$

which is the minimizer of $H(u)=\frac{1}{2}|| u-\widetilde{u}\left\|_{2}^{2}+\frac{\tau}{2}\right\| A u-d \|_{2}^{2}$. Its derivative is

$$
H^{\prime}(u)=(u-\widetilde{u})+\tau A^{*}(A u-d)
$$

and the minimizer $u^{k+1}$ verify Euler-Lagrange equation

$$
H^{\prime}\left(u^{k+1}\right)=0 \Longleftrightarrow\left(I+\tau A^{*} A\right) u^{k+1}=\widetilde{u}+\tau A^{*} d
$$

Assuming we know how to invert $I+\tau A^{*} A$, an immediate solution of (35) follows. As the computation of section 1.2 can be generalized to the discrete case, $A$ is here a Vandermonde hypermatrix, and thus $A^{*} A$ a Toeplitz hypermatrix. Therefore, the operator $A^{*} A$ is a non-periodic convolution with the point spread function $k$. This property was first used by Kostov for the inversion of the Radon transform alone [27] and by Feichtinger, Gröchenig and Strohmer for the restoration of irregularly sampled images [15]. To be able to use the Fast Fourier Transform (FFT), we make here the assumption that the non-periodic convolution can be approximated by a periodic convolution. It is however justified by the physical properties of an EPR acquisition: the sinogram on the border of the acquisition area is an almost null signal. 
Thanks to the convolution theorem, equation (35) is equivalent in the Fourier space to

$$
\begin{aligned}
& u^{k+1}+\tau k \star u^{k+1}=\widetilde{u}+\tau A^{*} d \Longleftrightarrow \quad(1+\tau \widehat{f}) \widehat{u^{k+1}}=\widehat{\widetilde{u}}+\tau \widehat{A^{*} g} \\
& \Longleftrightarrow \quad \widehat{u^{k+1}}=\frac{\widehat{\widetilde{u}}+\tau \widehat{A^{*} d}}{1+\tau \widehat{f}}
\end{aligned}
$$

2.2.2. TV-Curvelets model To implement the TV-Curvelets model in step (a) of the minimization algorithm, one needs to adapt the result of equation (32) for the dual variable $(p, w)$. The computation of the resolvent $\left(p^{k+1}, w^{k+1}\right)=$ $\left(I+\sigma \partial g^{\star}\right)^{-1}(\widetilde{p}, \widetilde{w})$ consists of projecting $(\widetilde{p}, \widetilde{w})=\left(p^{k}+\sigma \lambda_{1} \nabla v_{1}^{k}, w^{k}+\sigma \lambda_{2} W v_{2}^{k}\right)$ onto the set

$$
\left\{(p, w):\left(p_{1}[i, j]\right)^{2}+\left(p_{2}[i, j]\right)^{2} \leqslant 1 \text { and }\left|w_{r, s, t}\right| \leqslant 1\right\}
$$

Step (b) involves $K^{*}(p, w)=\left(-\lambda_{1} \operatorname{div}(p), \lambda_{2} W^{*} u_{2}\right)$ which is the adjoint operator of $K(u)=\left(\lambda_{1} \nabla u_{1}, \lambda_{2} W u_{2}\right)$. Thanks to the tight frame property of the curvelet transform described in [12], the adjoint of $W$ is equal to the inverse curvelet transform $W^{-1}$, thus yielding $K^{*}(p, w)=\left(-\lambda_{1} \operatorname{div}(p), \lambda_{2} W^{-1} u_{2}\right)$.

The computation of the resolvent $(I+\tau \partial F)^{-1}$ is similar to the resolution of equation (33), except that the variable $u$ is now equal to $\left(u_{1}, u_{2}\right)$ instead. In this case, the Euler-Lagrange equation is

$$
\left(\begin{array}{cc}
I+\tau A^{*} A & \tau A^{*} A \\
\tau A^{*} A & I+\tau A^{*} A
\end{array}\right)\left(\begin{array}{c}
u_{1} \\
u_{2}
\end{array}\right)=\left(\begin{array}{c}
\widetilde{u_{1}}+\tau A^{*} d \\
\widetilde{u_{2}}+\tau A^{*} d
\end{array}\right)
$$

with $\widetilde{u}=u^{k}-\tau K^{*}\left(p^{k+1}, w^{k+1}\right)$.

Again, by considering operator $A^{*} A$ as a convolution with the point spread function $k$ and computing it in the Fourier space, the resolution of system 37 yields

$$
\widehat{u_{1}}=\frac{(1+\tau \widehat{k}) \widehat{v_{1}}-\tau \widehat{k} \widehat{v_{2}}}{1+2 \tau \widehat{k}} \quad \text { and } \quad \widehat{u_{2}}=\frac{(1+\tau \widehat{k}) \widehat{v_{2}}-\tau \widehat{k} \widehat{v_{1}}}{1+2 \tau \widehat{k}}
$$

with

$$
\left(\begin{array}{l}
v_{1} \\
v_{2}
\end{array}\right)=\left(\begin{array}{l}
\widetilde{u_{1}}+\tau A^{*} d \\
\widetilde{u_{2}}+\tau A^{*} d
\end{array}\right)
$$




\section{Results and discussion}

Numerical simulations are processed with MATLAB [2]. Discrete Fourier transforms are computed using FFTW [17]. When computation is required on a polar raster, we use the NUFFT [20]. The curvelet part requires the package described in [12]. Real data are loaded using Easyspin [38].

\subsection{Simulated data}

3.1.1. Robustness to few angle acquisitions First tests and simulations have been conducted on the Shepp-Logan phantom introduced in [37]. Figure 1a presents the Shepp-Logan phantom. Figure $1 \mathrm{~b}$ presents the spectrum $h$, simulated as a derivative of a gaussian distribution. Figure $1 \mathrm{c}$ is the simulated sinogram $S$, created as the Radon transform of the Shepp-Logan transform, implemented with MATLAB's function radon with 100 angles and then convoluted with the spectrum $h$. Both spectrum and sinogram are then corrupted with a gaussian noise of variance $\sigma=0.03$ proportionally to their amplitudes.

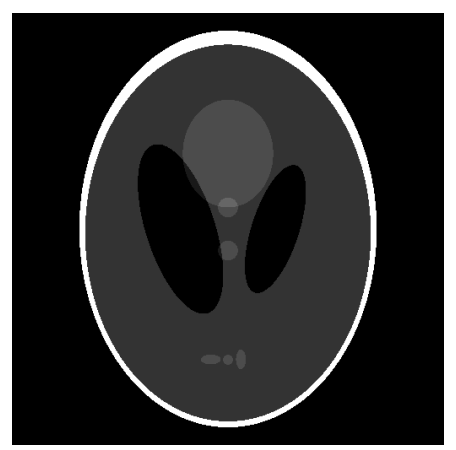

(a) Shepp-Logan phantom

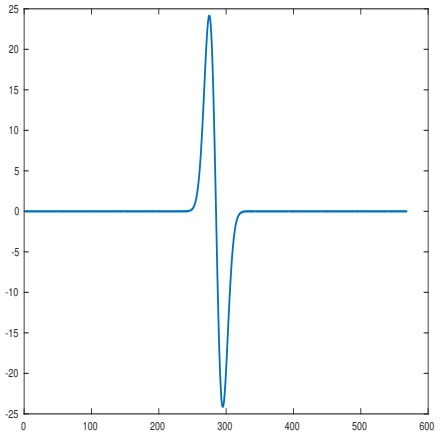

(b) Simulated spectrum

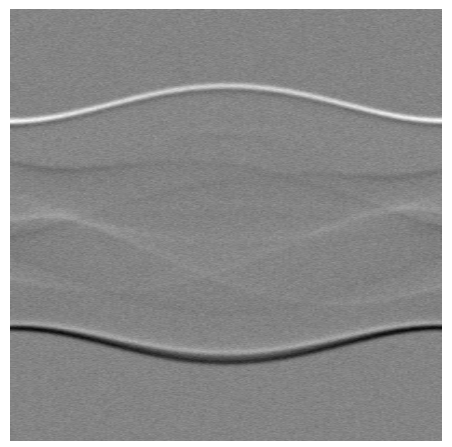

(c) Sinogram

Figure 1: Simulated EPRI data on Shepp-Logan phantom

Our model with a TV regularization is compared to the reconstruction algorithm implemented in Xepr [1], the commercial software provided by Bruker with EPRI devices. It consists of a deconvolution step followed by a backprojection step.

Figure 2 presents the reconstruction results of the two methods applied to an input data admitting a decreasing number of acquisition angles. The TV reconstructions are computed with the parameter $\lambda=0.05$. The Xepr 
deconvolution is made with a gaussian window of width 0.1 and threshold 0.5. The backprojection step is made with the parameters Reduce $=512$ and ImageSize $=512$.

The proposed TV model outperforms the Xepr model in figure 2a both visually: the TV reconstruction is sharp whereas the Xepr image is noisy, and quantitatively: the PSNR of the TV reconstruction compared to the original phantom is 18.6, whereas it is only 16.4 for the Xepr image. As the number of angles decreases significantly in figures $2 \mathrm{~b}$ and $2 \mathrm{c}$, the Xepr reconstruction admits visible artifacts whereas the TV reconstruction is very robust to the lack of information in between acquisition lines. In figure 2c, the PSNR or the TV model is 15.4 where it is only 10.9 for the Xepr image. This numerical experiment thus validates our first model with the total variation as a regularization for simulated sinograms of piecewise-constant images.

Because the acquisition time of an EPRI device is a strong limitation for in vivo measurements, the ability to correctly reconstruct an image from a small number of acquisition lines is a real improvement for small animals and human EPR imaging.

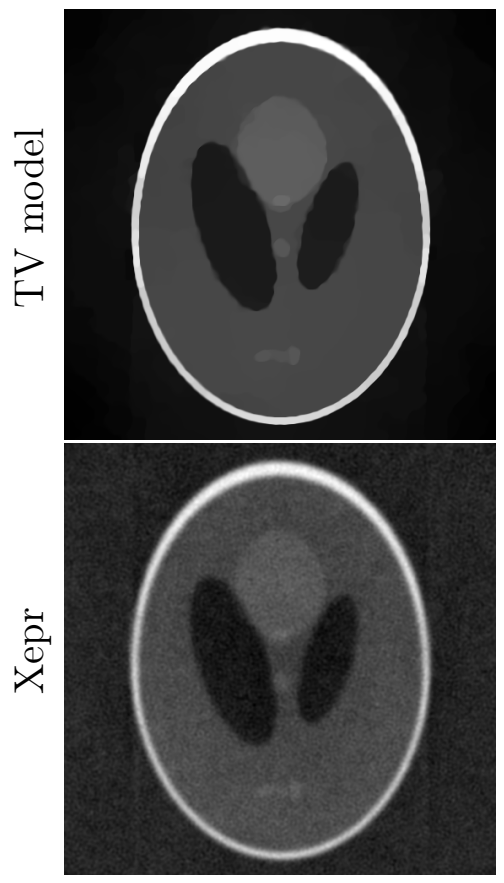

(a) 100 angles
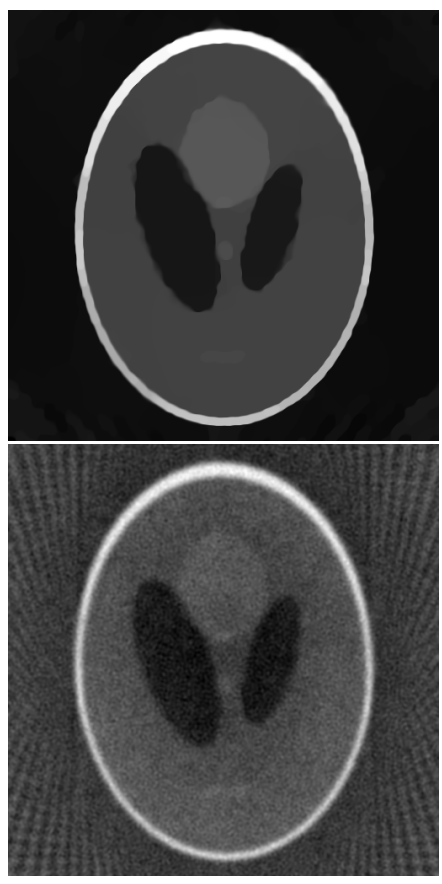

(b) 50 angles

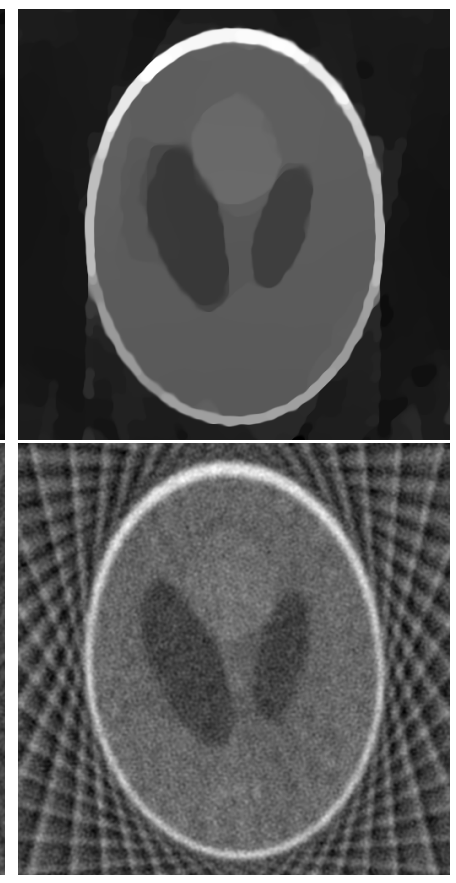

(c) 20 angles

Figure 2: Reconstructions with a decreasing number of acquisition angles. 
3.1.2. Retrieval of simulated textures The TV model has been validated on acquisitions with very few angles. This section justifies the need for a second choice of regularization for textured data: the TV-Curvelets model. Henceforth, some texture of various kinds has been added to the phantom of Shepp-Logan. Input simulated data are shown in figure $3 \mathrm{a}$, along with their reconstruction with the TV model in figure $3 \mathrm{~b}$ with parameter $\lambda=10^{-2}$.

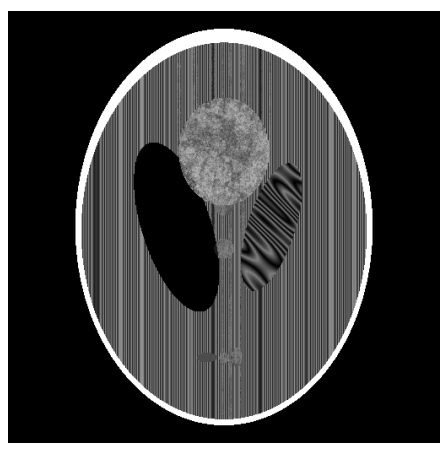

(a) Original

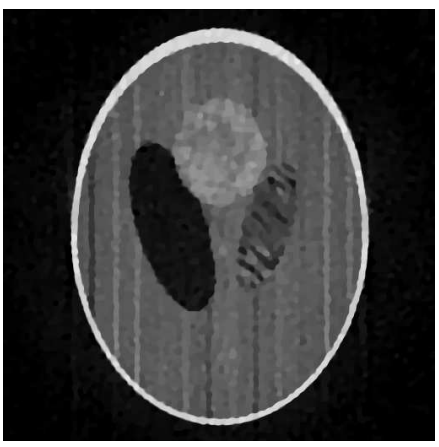

(b) Reconstruction

Figure 3: Reconstruction of textured Shepp-Logan with TV model

Although the global shape of the phantom is retrieved, the details of the textures are lost through the reconstruction process. This is due to the simplicity of the TV model. Therefore, a more sophisticated model is applied in order to retrieve the strongly oscillating textures : the TV-Curvelets model, described in section 1.3.2 with parameters $\lambda_{1}=5.10^{-2}$ and $\lambda_{2}=5.10^{-5}$.

Results are shown in figure 4 and a zoomed comparison of the TV vs TVCurvelets model stands in figure 5 . Figure $5 \mathrm{c}$ visually shows a finer reconstruction of the details of the textures than in figure $5 \mathrm{~b}$. 


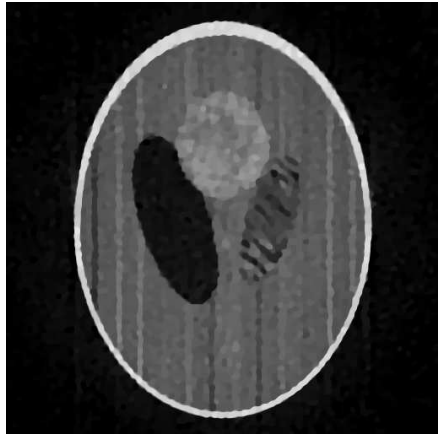

(a) Reconstruction

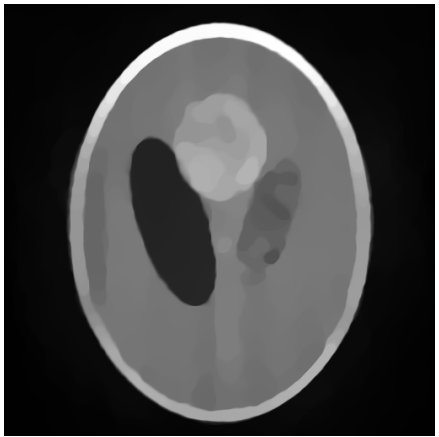

(b) Cartoon part $u_{1}$

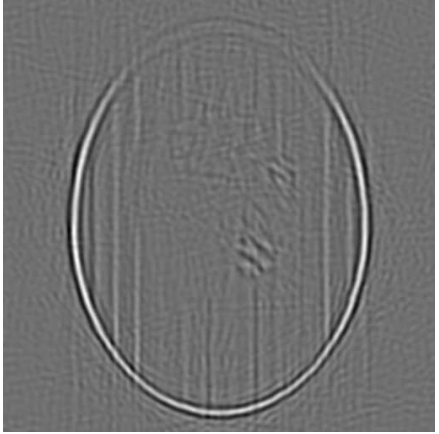

(c) Textured part $u_{2}$

Figure 4: Reconstruction with TV-Curvelets model

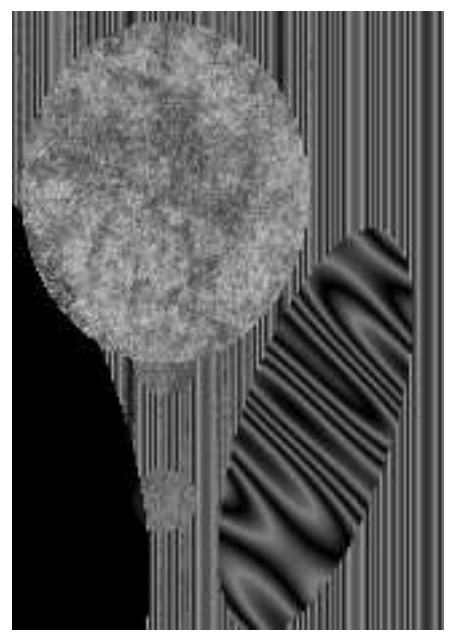

(a) Original

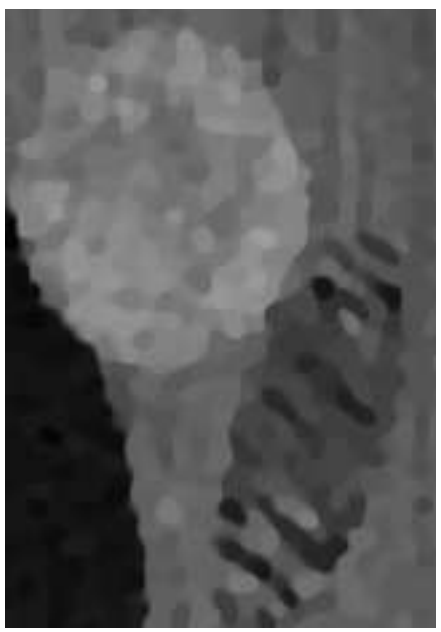

(b) TV model

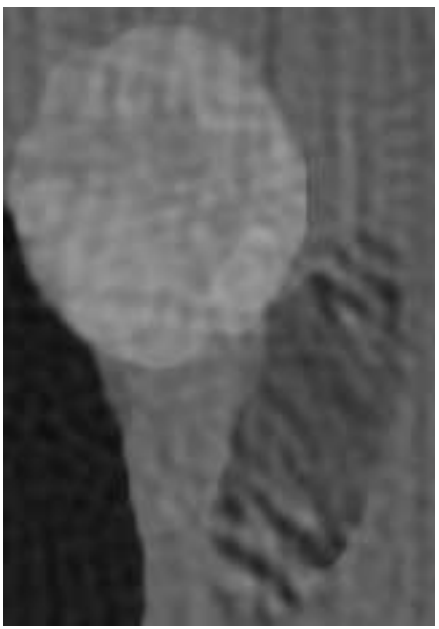

(c) TV-Curvelets model

Figure 5: Details of the Shepp-Logan phantom with texture

\subsection{Experimental data}

Further tests were conducted on real experimental data. The sample was an irradiated distal phalanx kindly furnished by Philippe Lévèque from REMA, Louvain La Neuve University, Bruxelles. Irradiation of solid part in the body leads to paramagnetic defaults which can be used for retrospective irradiation dosimetry and are observable using EPRI [26].

A picture of the sample is shown on figure $6 \mathrm{~b}$ and a slice of a CT scan with the same orientation is shown on figure $6 \mathrm{~b}$. Micro-CT imaging was obtained using 
a 1178 X-ray Skyscan computed tomograph with settings at $50 \mathrm{kV}, 615 \mathrm{~mA}$, pixel size $80 \mu \mathrm{m}$. Raw micro-CT images were processed using the Skyscan software suite Nrecon, CTAn, and CTVol.

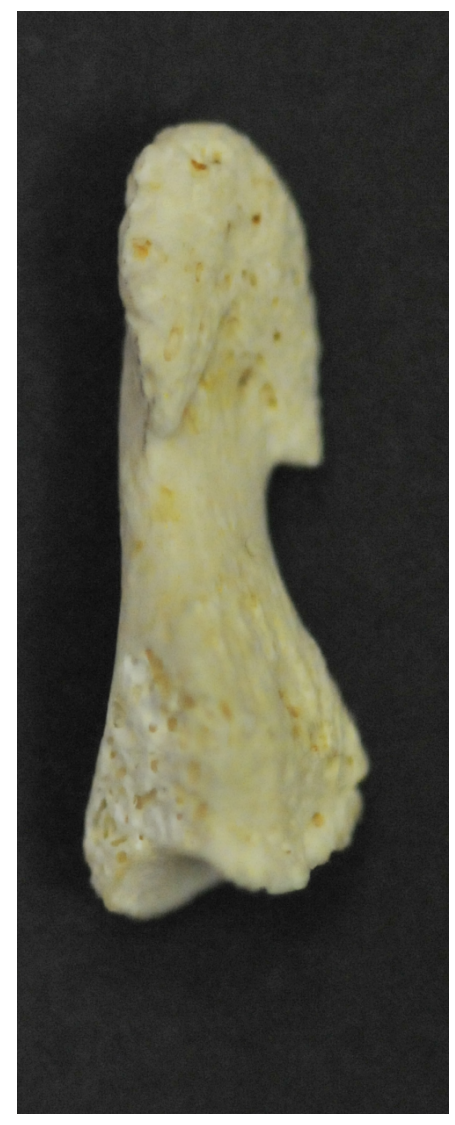

(a) Picture

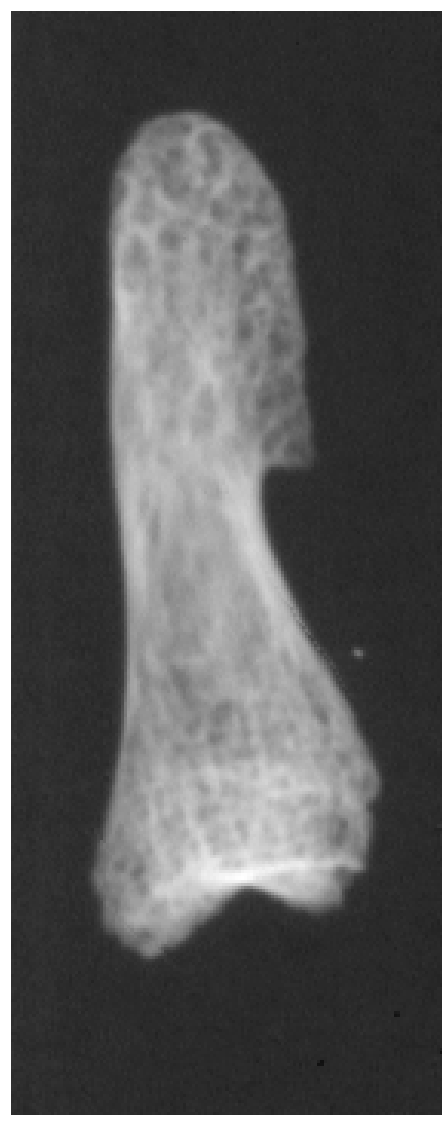

(b) Micro-CT scan

Figure 6: Irradiated phalanx

3.2.1. Long acquisition time An EPRI acquisition was recorded at $10 \mathrm{GHz}$ using a X-Band Bruker E540 spectrometer fitted with a TMS resonator. The settings of the device were $0.3 \mathrm{mT}$ for the amplitude of field modulation and $100 \mathrm{kHz}$ for its frequency, a conversion time of $163.84 \mathrm{~ms}$ and an identical time constant. The X-band EPR images were obtained using $1.75 \mathrm{~T} / \mathrm{m}$ gradient strength, with 117 tomographic projections on theta angle to obtain a 2D image. The whole experiment lasted 11 hours. The obtained spectrum and sinogram are shown on figure 7 . 


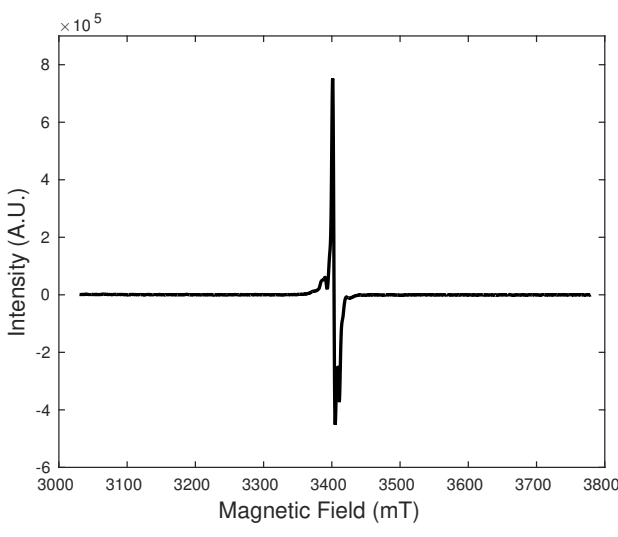

(a) Spectrum

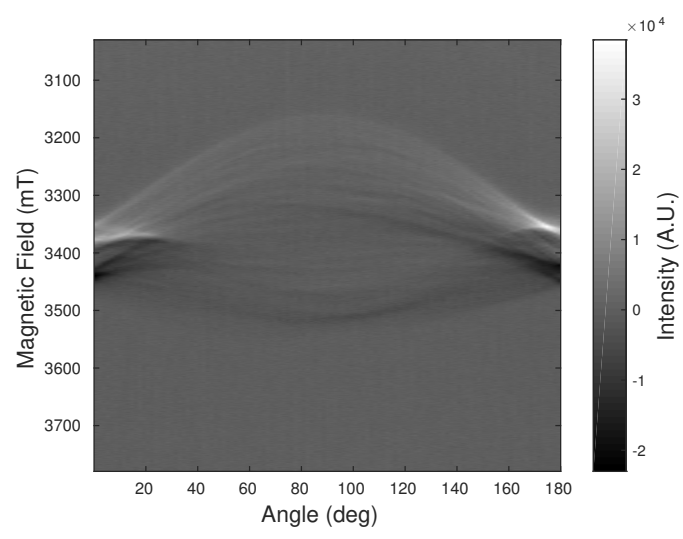

(b) Sinogram

Figure 7: Acquisition data on the phalanx

Figure 8a presents the reconstruction with the TV-Curvelets model. The associated decomposition in cartoon and texture parts is shown in figures $8 \mathrm{~b}$ and $8 \mathrm{c}$ respectively. Due to the high amplitude of the data compared to the SheppLogan simulation, the parameters were set to $\lambda_{1}=10^{9}$ and $\lambda_{2}=10^{6}$. Many textures are retrieved in the reconstruction, as expected after the simulations on the Shepp-Logan phantom.

For comparison, reconstructions made with Xepr and TV model are shown in figure 9 . The parameter $\lambda$ was set to $10^{12}$. As shown by the results obtained on simulated data, the TV model outperforms Xepr, especially regarding the noise level, but does not reconstruct the textures enough to take into account some details of the phalanx. Subjective evaluation yields a clear preference for the TV-Curvelets model.

Furthermore, the small details of the texture retrieved by the TV-Curvelets model in figure $9 \mathrm{c}$ are coherent with the ones visible on the $\mathrm{CT}$ scan of the phalanx in figure 6b. It means that the effects of the curvelet regularization did not create false texture, but instead retrieved existing textures. Because the CT is an anatomic imaging device whereas the EPRI is a molecular imaging device, we can assume that the lasting effects of the irradiation is correlated with the anatomic map of the bones. Those similarities strongly validate our TV-Curvelets model. 


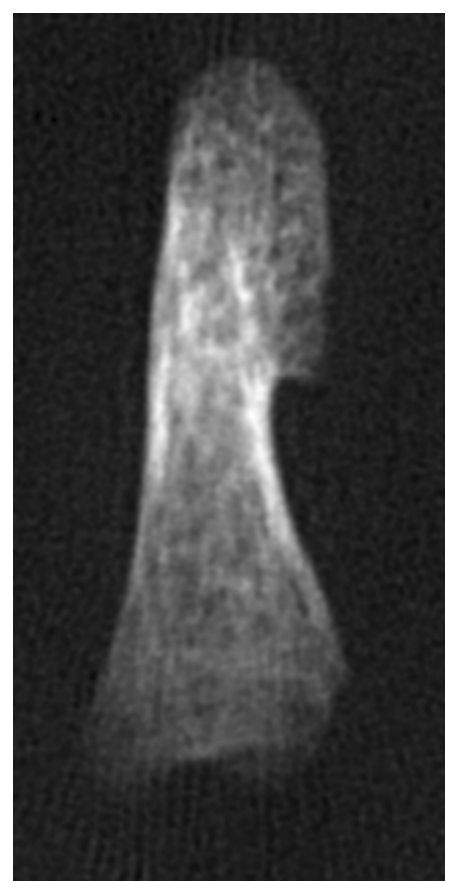

(a) Reconstruction

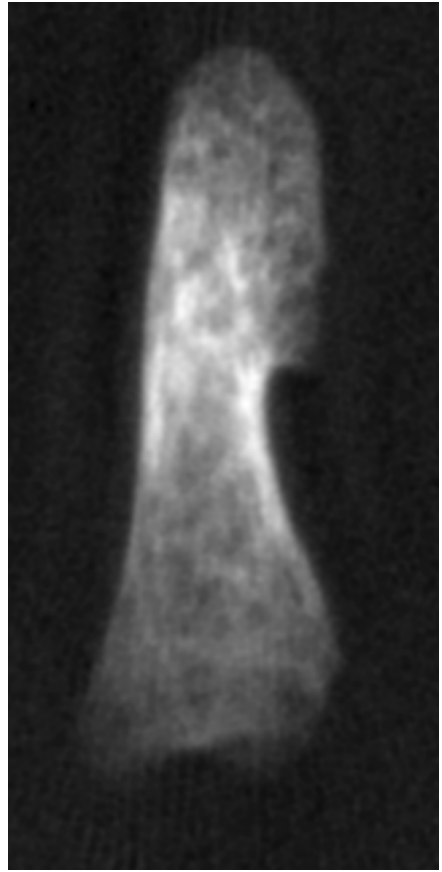

(b) Cartoon part $u_{1}$

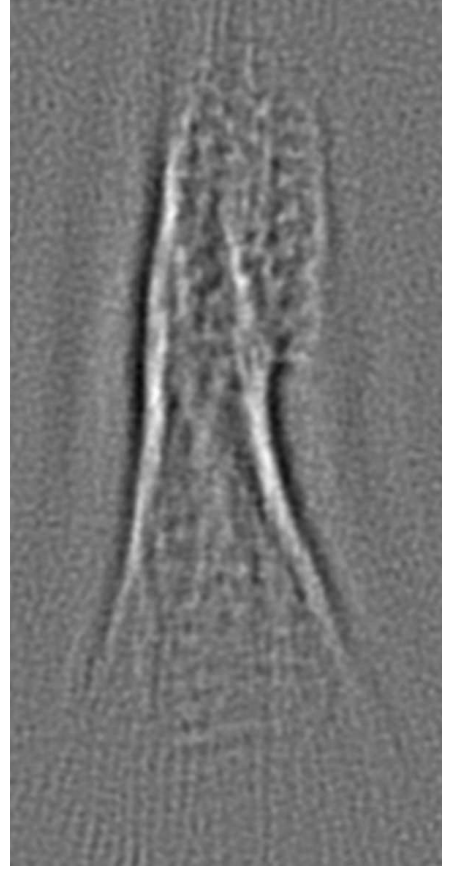

(c) Textured part $u_{2}$

Figure 8: Reconstruction with TV-Curvelets model

3.2.2. Reduction of the experimental data As the acquisition of the sinogram of the phalanx lasted 11 hours, reconstructions were conducted on the same sinogram by taking only a fifth of the projection angles and a quarter of the acquisition points, thus mimicking an acquisition time reduced by a factor 20 . The parameters were adapted to $\lambda_{1}=5.10^{7}$ and $\lambda_{2}=10^{5}$. Reconstructions are shown in figure 10. Although the textures do not appear as clearly as with the whole set of data, the TV-Curvelets model succeeds in retrieving the global shape of the phalanx, whereas Xepr fails to reconstruct the border of the phalanx. Therefore, the TVCurvelets model proves its efficiency for preliminary acquisitions, when the zone on which to concentrate the longer acquisition has to be specified. 


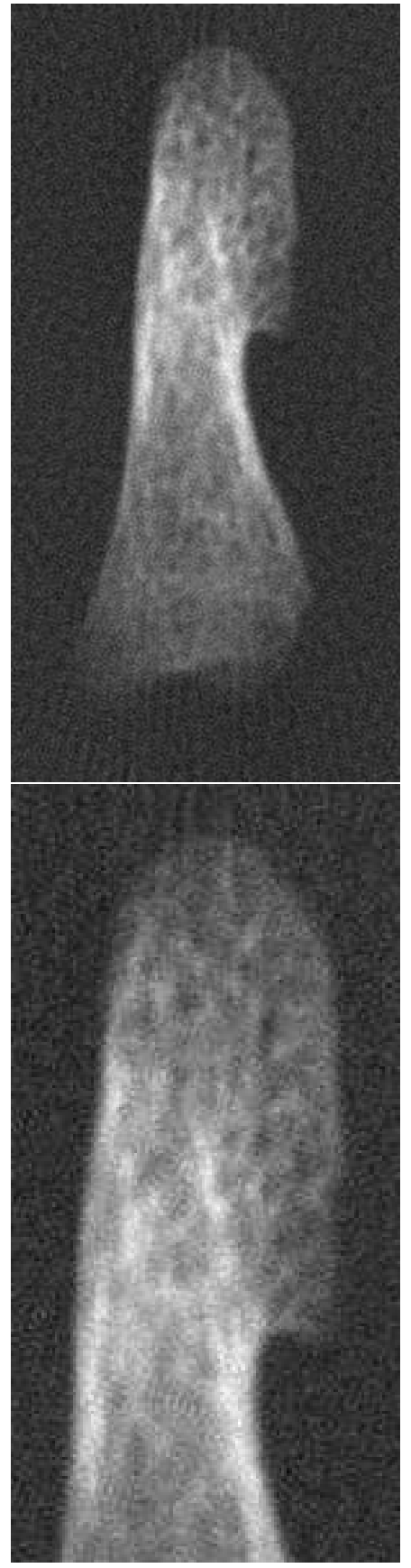

(a) Xepr
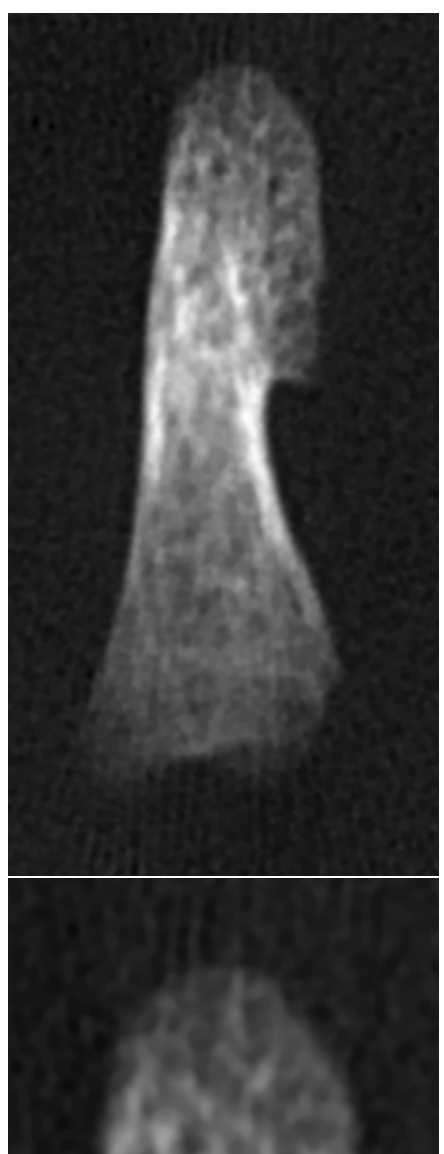

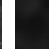

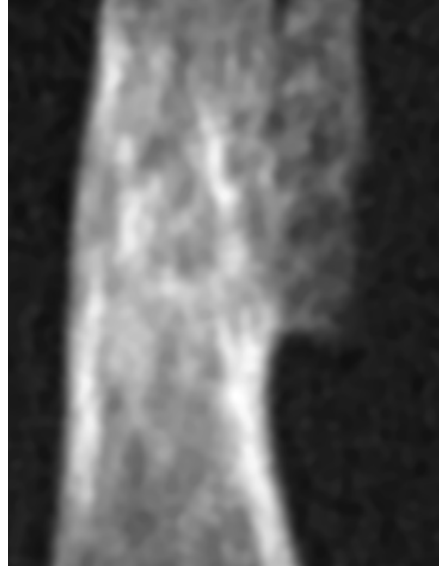

(b) TV model
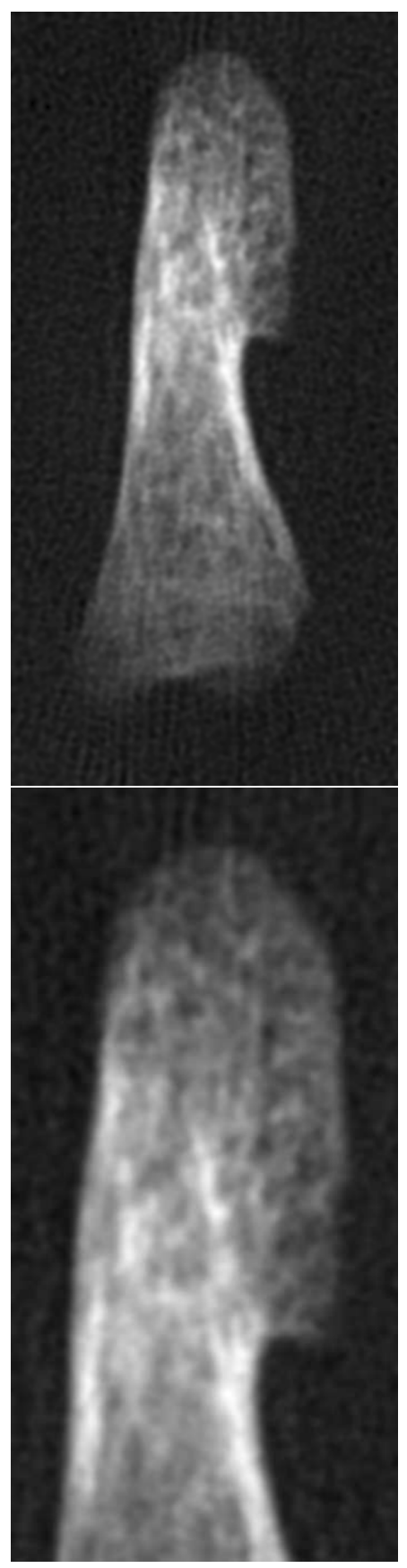

(c) TV-Curvelets model

Figure 9: Three reconstruction models and zoom 


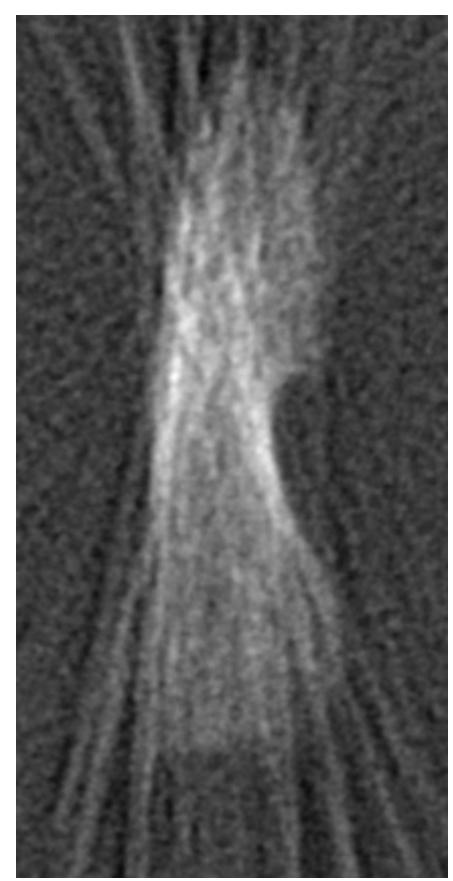

(a) Xepr

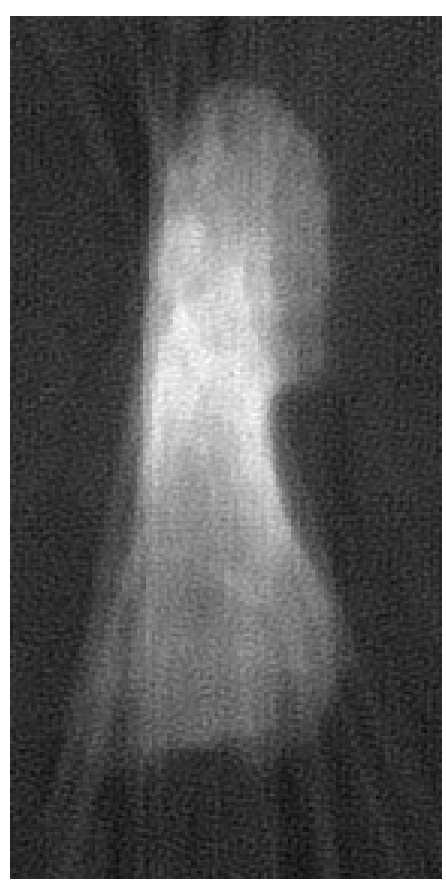

(b) TV-Curvelets

Figure 10: Reconstruction with reduced data

\section{Conclusion}

Our contribution to EPRI is a thorough analysis of the direct model. It leads to properties that allow us to efficiently minimize an objective function specially designed for the EPRI problem. The proposed algorithm is fast and admits a proof of convergence towards the minimizer of the objective function.

Our choices regarding the data-fidelity term and regularization term are validated by simulated experiments. When no texture is present in the image, the TV model outperforms the classic Xepr reconstruction. And the TV-Curvelets model turns out to be very satisfying when applied to real acquisition data: the curvelet regularization retrieves textures that strongly match the CT anatomic image of the phalanx bone, where both Xepr and our simpler model with the TV regularization do not provide as much details. 


\section{Acknowledgments}

The EPR data in this manuscript were obtained using equipment supported jointly by the French National Ministry of Research (PPF IRPE), the "Fondation pour la Recherche Médicale" (FRM DGE20061007745), the CNRS (Department of Chemistry and Life Sciences). Imaging was conducted on the Imaging Life Platform (Plateforme d'Imageries du Vivant) of University Paris Descartes and Research Infrastructure CNRS FR 3443 RENARD. The PhD of Maud Kerebel is founded by a grant double-culture by University Sorbonne Paris Cité. The authors would like to thank Philippe Lévèque and Bernard Gallez (REM, Bruxelles) for having provided the phalanx, Rémy Abergel for the discussions regarding the minimization algorithms, and Sonia Lajnef for her constant support regarding the EPRI acquisitions.

\section{References}

[1] Xepr, version 2.6b.36. Bruker, 2009. https://www . bruker.com/fr/products/mr/epr/epr-software/epr-software/xepr.

[2] MATLAB and Image Processing Toolbox Release 2015a. The MathWorks, Inc., Natick, Massachusetts, United States.

[3] The Mathematics of Computerized Tomography, Ill-posedness and Accuracy, chapter 4.

[4] R. Ahmad, B. Clymer, Y. Deng, G. He, D. Vikram, P. Kuppusamy, and J. Zweier. Optimization of data acquisition for EPR imaging. Journal of Magnetic Resonance, 2006.

[5] R. Ahmad, B. Clymer, D. Vikram, Y. Deng, H. Hirata, J. Zweier, and P. Kuppusmy. Enhanced resolution for EPR imaging by two-step deblurring. Journal of Magnetic Resonance, 2006.

[6] J.-F. Aujol and A. Chambolle. Dual norms and image decomposition models. International Journal of Computer Vision, 2005.

[7] L. Binet, D. Gourier, and S. Derenne. Potential of EPR imaging to detect traces of primitive life in sedimentary rocks. Earth and Planetary Science Letters, 2008.

[8] A. Blank, J. Freed, N. Kumar, and C. Wang. Electron spin resonance microscopy applied to the study of controlled drug release. Journal of Controlled Release, 2006.

[9] A. Chambolle. An algorithm for total variation minimization and applications. Journal of Mathematical Imaging and Vision, 2004.

[10] A. Chambolle and T. Pock. A first-order primal-dual algorithm for convex problems with applications to imaging. Journal of Mathematical Imaging and Vision, 2011.

[11] S. Deans. The Radon Transform and Some of Its Applications. Krieger Publishing Company, 1983.

[12] D. Donoho E. Candès, L. Demanet and L. Ying. Fast discrete curvelet transforms. SIAM, 2006. 
[13] M. Sathiya et al. Electron paramagnetic resonance imaging for real-time monitoring of Li-ion batteries. Nature Communications, 2015.

[14] N. Bezières et al. First combined in vivo X-Ray tomography and high-resolution molecular electron paramagnetic resonance (EPR) imaging of the mouse knee joint taking into account the disappearance kinetics of the EPR probe. Molecular Imaging, 2012.

[15] H. Feichtinger, K. Gröchenig, and T. Strohmer. Efficient numerical methods in non-uniform sampling theory. Numerische Mathematik, 1995.

[16] Y. Frapart. In vivo electron paramagnetic resonance and imaging in biomedical science. Advances in Bio-Imaging, 2012.

[17] M. Frigo and S. Johnson. The design and implementation of FFTW3. Proceedings of the IEEE, 2005.

[18] F. Frühauf, C. Pontow, and O. Scherzer. Texture enhancing based on variational image decomposition. Mathematical Image Processing, 2011.

[19] H. Fujii, H. Sato-Akaba, K. Kawanishi, and H. Hirata. Mapping of redox status in a brain-disease mouse model by three-dimensional EPR imaging. Magnetic Resonance in Medicine, 2011.

[20] L. Greengard and J.-Y. Lee. Accelerating the Nonuniform Fast Fourier Transform. SIAM, 2004

[21] D. Hayner. The missing cone problem in computer tomography and a model for interpolation in synthetic aperture radar. PhD thesis, University of Illinois, 1983.

[22] G. Herman. Fundamentals of Computerized Tomography, Image Reconstruction from Projections. Advances in Pattern Recognition, Springer, 2009.

[23] H. Hirata and H. Fujii. Free radicals in living systems: In vivo detection of bioradicals with EPR spectroscopy. Current Organic Chemistry, 2006.

[24] C. Johnson, D. McGarry, J. Cook, N. Devashayam, J. Mitchell, S. Subramanian, and M. Krishna. Maximum entropy reconstruction methods in electron paramagnetic resonance imaging. Annals of Operations Research, 2003.

[25] D. Johnson, R. Ahmad, G. He, A. Samouilov, and J. Zweier. Compressed sensing of spatial electron paramagnetic resonance imaging. Magnetic Resonance in Medecine, 2014.

[26] N. Khan, B. Williams, and H. Swartz. Clinical applications of in vivo EPR: Rationale and initial results. Applied magnetic Resonance, 2006.

[27] C. Kostov. Toeplitz structure in slant-stack inversion. Society of Exploration Geophysicists, 1990.

[28] P. Kuppusamy and J. Zweier. Cardiac applications of EPR imaging. NMR in Biomedicine, 2004.

[29] K. Mader, H. Swartz, R. Stosser, and H. Borchert. The application of EPR spectroscopy in the field of pharmacy. Pharmazie, 1994.

[30] Y. Meyer. Wavelets and operators, volume 37 of Cambridge Studies in Advanced Mathematics. Cambridge University Press, Cambridge, 1992.

[31] J. Palmer, L. Potter, D. Johnson, J. Zweier, and R. Ahmadb. Dual-scan acquisition for accelerated continuous-wave EPR oximetry. Journal of magnetic Resonance, 2013.

[32] X. Pan, E. Sidky, and M. Vannier. Why do commercial CT scanner still employ traditional, filtered back-projection for image reconstruction? Inverse Problems, 2009.

[33] P. Plonka. Electron paramagnetic resonance as a unique tool for skin and hair research. 
Experimental Dermatology, 2009.

[34] Z. Qiao, G.Redler, B. Epel, Y. Qian, and H. Halpern. 3D pulse EPR imaging from sparse-view projections via constrained total variation minimization. Journal of Magnetic Resonance, 2015.

[35] J. Radon. Über die Bestimmung von Funktionen durch ihre Integralwerte längsgewisser Mannigfaltigkeiten. Berichte über die Verhandlungen der Königlich-Sächsischen Gesellschaft der Wissenschaften zu Leipzig, 1917.

[36] L. Rudin, S. Osher, and E. Fatemi. Nonlinear total variation based noise removal algorithms. Physica D, 1992.

[37] L. Shepp and B. Logan. The Fourier reconstruction of a head section. IEEE Transactions on Nuclear Science, 1974.

[38] S. Stoll and A. Schweiger. Easyspin, a comprehensive software package for spectral simulation and analysis in EPR. Journal of Magnetic Resonance, 2006.

[39] M. Tseitlin, T. Czechowski, S. Eaton, and G. Eaton. Regularized optimization (RO) reconstruction for oximetric EPR imaging. Journal of magnetic Resonance, 2008.

[40] M. Tseitlin, A. Dhami, S. Eaton, and G. Eaton. Comparison of maximum entropy and filtered back-projection methods to reconstruct rapid-scan EPR images. Journal of Magnetic Resonance, 2007.

[41] S. Velan, R. Spencer, J. Zweier, and P. Kuppusamy. Electron paramagnetic resonance oxygen mapping (EPROM): direct visualization of oxygen concentration in tissue. Magnetic resonance in medicine, 2000.

[42] L. Vese and S. Osher. Modeling textures with total variation minimization and oscillating patterns in image processing. Journal of Scientific Computing, 2002.

[43] J. Weil and J. Bolton. Electron Paramagnetic Resonance: Elementary Theory and Practical Applications, Second Edition. Wiley, 2007. 